\section{Responses to selinexor in multiple myeloma}

Virtually no treatment options are available for patients with multiple myeloma (MM) refractory to proteasome inhibitors, immunomodulatory agents and monoclonal antibodies (triple-class refractory MM). Now, responses to selinexor, a novel selective inhibitor of exportin 1 (which is involved in the nuclear export of tumour suppressor proteins and is overexpressed in MM), have been reported in this setting.

In the single-arm phase II STORM trial, 122 patients with MM who had previously received bortezomib, carfilzomib, lenalidomide, pomalidomide, daratumumab and an alkylating agent were treated with selinexor plus low-dose dexamethasone. A total of 32 patients (26\%) had a partial response (PR) or better, including 2 stringent complete responses, 6 very good PRs and 24 PRs; in total, 48 patients (39\%) had a minimal response or better. Median progression-free survival and overall survival (OS) durations were 3.7 months and 8.6 months, respectively. The subgroup of patients with a PR or better and those with a minimal response or better both had median OS durations of 15.6 months.
The most common grade 3-4 adverse events (AEs) were thrombocytopenia (in $59 \%$ of patients), anaemia (44\%), hyponatraemia (22\%) and neutropenia (21\%). A total of 12 patients died from AEs, which were considered treatment-related in a patient with pneumonia and another one with sepsis.

Analysis of samples from 35 patients led to the identification of a four-protein classifier with high predictive performance (area under the curve 0.86). A predictive accuracy of $83 \%$ was observed in a validation set with samples from 12 patients.

The activity of selinexor in various combinations is being investigated in several comparative trials involving patients with MM. The publication of these results will enable to determine whether or not selinexor should be incorporated in the routine management of patients with MM.

Diana Romero

ORIGINAL ARTICLE Chari, A. et al. Oral selinexordexamethasone for triple-class refractory multiple myeloma. N. Engl.J. Med. 381, 727-738 (2019)

\title{
DYNAMIC insights into MRD responses early after resection of NSCLC
}

Measurable residual disease (MRD) analysis using circulating cell-free tumour DNA (ctDNA) has promising prognostic value. However, few studies have prospectively evaluated ctDNA dynamics associated with tumour resection and the optimal time point of MRD testing. New insights into these aspects come from the DYNAMIC study.

In DYNAMIC, 205 consecutive patients with resectable suspected non-small-cell lung cancer (NSCLC) underwent ctDNA analysis of plasma samples collected prospectively at prespecified time points immediately before, during $(5 \mathrm{mins}, 30 \mathrm{mins}$ and $2 \mathrm{~h}$ after tumour resection) and after surgery ( 1 day, 3 days and 1 month). Ultimately, 26 of 36 patients with detectable ctDNA in pre-surgery samples were included in the study.

Average variant allele frequencies prior to surgery and at the three time points during surgery were $2.72 \%, 2.11 \%, 1.14 \%$ and $0.17 \%$, respectively. The median ctDNA half-life was 35 mins, suggesting that ctDNA analysis can provide a near real-time readout of tumour burden. At day 1 after surgery, 12 patients had MRD, 6 of whom had subsequent conversion to MRD-negative status. Accordingly, MRD positivity at day 1 was not predictive of relapse-free survival (RFS; HR $1.33,95 \% \mathrm{Cl} 0.38-4.60 ; P=0.66$ ) nor overall survival (OS; HR 1.96, 95\% Cl 0.33-11.72; $P=0.46)$. By contrast, MRD positivity at day 3 was predictive of unfavourable RFS (HR 7.55, 95\% Cl 2.09-27.27; $P=0.002$ ) and OS (HR 14.22, 95\% Cl 1.58-128.15; $P=0.018)$; very similar correlations were observed with MRD positivity at 1 month.

The ability to predict outcomes according to MRD status at a time point before patient discharge might facilitate the development of personalized care plans. However, ctDNA detection in a larger proportion of patients with early stage cancers is required to improve the clinical utility of this approach.

David Killock

ORIGINAL ARTICLE Chen, K. et al. Perioperative dynamic changes in circulating tumor DNA in lung cancer patients (DYNAMIC). Clin. Cancer Res. https://doi.org/10.1158/1078 0432.CCR-19-1213 (2019)

\section{CD19 affinity - is lower also better?}

Most patients with $\mathrm{CD} 19^{+}$treatmentrefractory acute lymphoblastic leukaemia (ALL) respond to anti-CD19 chimeric antigen receptor (CAR) T cells. Nonetheless, adverse events can limit the utility of this approach. Now, data from a phase I study indicate that CAR T cells with a novel single-chain variable fragment (scFv) with a lower affinity for CD19 might overcome these limitations.

Researchers generated novel anti-CD19 scFv fragments using a CAT131E10 hybridoma (CAT) and conducted initial in vitro comparisons of this scFv versus the FMC63 scFv, which is included in the FDA-approved CAR T cell therapy tisagenlecleucel. CAT scFvs were found to have a 40-fold lower affinity for CD19 than the FMC63 scFv, largely owing to a much faster rate of antigen dissociation. CAT-CAR T cells also had greater levels of in vitro antigen-specific toxicity and proliferation, and these findings were confirmed in vivo.

The safety and efficacy of CAT-CAR T cells was then investigated in 17 patients with high-risk treatment-refractory CD19+ $\mathrm{ALL}$. Among 14 patients who received an infusion, 12 had a molecular complete response after 3 months (overall response rate $71 \%$ ). The expansion of CAT-CAR T cells was approximately threefold higher than that reported for tisagenlecleucel at 28 days. The 1-year overall survival and event-free survival was $63 \%$ and $46 \%$, respectively. CAT-CAR T cells were detectable at the latest follow-up appointment in 11 of 14 patients.

Notably, no patient required tocilizumab or intensive care for grade $\geq 3$ cytokinerelease syndrome (CRS), although 3 patients required symptom management for CRS-related hypotension. Similarly, only one patient had a grade $\geq 3$ neurotoxicity (leukoencephalopathy) and this was attributed to use of fludarabine for lymphodepletion. Grade $\geq 3$ haematological toxicities, however, were common, including neutropenia in six patients and thrombocytopenia in three.

These data suggest that a lower-affinity anti-CD19 CAR enables robust expansion, with fewer serious adverse events.

These findings warrant further evaluation.

Peter Sidaway

ORIGINAL ARTICLE Ghorashian, S. et al. Enhanced CAR T cell expansion and prolonged persistence in pediatric patients with ALL treated with a low-affinity CD19 CAR. Nat. Med. 25, 1408-1414 (2019) 